\title{
Solutions for downslope pipeline walking on a seabed with a peaky tri-linear soil resistance model
}

\author{
Adriano Castelo \\ PhD Candidate, The University of Western Australia \\ UWA (M053), 35 Stirling Highway CRAWLEY WA 6009, Australia \\ 21445298@student.uwa.edu.au \\ David White \\ Professor, The University of Southampton \\ Boldrewood Innovation Campus, Southampton, SO16 7QF, UK \\ david.white@soton.ac.uk

\section{Yinghui Tian} \\ Associate Professor, The University of Melbourne \\ Parkville, 3010, Australia \\ yinghui.tian@unimelb.edu.au
}

\begin{abstract}
Offshore pipelines used for transporting hydrocarbons are cyclically loaded by great variations of pressure and temperature. These variations can induce axial instability in such pipelines. This instability may cause the pipelines to migrate globally along their length; an effect known as pipeline walking. Traditional models of pipeline walking have considered the axial soil response as rigid-plastic (RP); however, such behaviour does not match observations from physical soil tests. It leads to inaccurate estimates of walking rate (WR) per cycle and over design. In this paper, a tri-linear (3L) soil resistance model is used to represent seabed resistance to investigate the behaviour of pipeline walking. Different parameters, i.e. shapes and properties of tri-linearity (within the peaky soil model type) have been considered leading to a closed-form solution. This solution improves understanding of the main properties involved in the peaky tri-linear soil behaviour by providing a set of analytical expressions for pipe walking, which were benchmarked and validated against a set of finite element analyses.
\end{abstract}

\section{KEYWORDS}

axial resistance; pipe-soil interaction; pipeline walking; finite-element modelling; offshore engineering 


\section{INTRODUCTION}

As offshore oil and gas industry increasingly explores deep water reservoirs,

3 offshore pipelines become progressively important. Under operational load cycles, they

4 expand and contract in response to temperature and pressure changes. However, these

5 expansion and contraction cycles may have an asymmetric behaviour due to seabed

6 slopes or other factors, such as multiphasic flow (Bruton et al., 2010), and thermal

7 transients (Carr et al., 2006). The asymmetric expansion and contraction directly impacts

8 the stability of these pipelines causing them to migrate in one direction, which generates

9 the phenomenon known as pipeline walking (Carr et al., 2003). Pipeline walking

10 increases cost and risk and may severely impact the subsea system (Tornes et al., 2000).

11 It may overstress connections, alter loads and strains in any engineered lateral buckle

12 and may also present the need for anchoring. Hence, accurately identifying and

13 estimating pipeline walking is necessary to decrease the risk of production loss and

14 environmental impact, and it can significantly decrease project development costs.

15 Presently, the common practice in the industry is to evaluate pipeline walking

16 during the design phase using a set of analytical formulations as per Bruton et al. (2010).

17 These calculations consider various aspects, such as operational (temperature, pressure,

18 etc.), environmental (seabed overall slope angle, soil friction coefficient, etc.) and

19 physical pipeline properties (length, steel wall thickness, etc.); but still imply some

20 limitations such as the idealised rigid-plastic pipe-soil interaction, and the single seabed

21 slope. Accurately evaluating high-temperature and high-pressure pipelines for

22 downslope pipeline walking is of paramount importance to the industry because these 
23 conditions are commonly found in fully operational areas, such as the Gulf of Mexico,

24 North Sea and Northwest Australia, as well as in frontier locations, which are still in early 25 stages of exploration, such as the Brazilian Pre-Salt and the Arctic Region. The analytical

26 formulation is based on an idealised soil resistance model and can provide inaccurate

27 walking rates. Then, the assessment requires further improvement of the soil resistance

28 model to overcome this limitation.

29 Costly and time demanding finite element analyses are used to confirm walking

30 behaviour and to generate a reliable walking rate. However, emerging academic

31 research (Castelo et al., 2019; Castelo et al., in press b) demonstrates that, if more

32 realistic soil behaviour is considered in the analytical formulae, the requirement for time

33 demanding and expensive finite element analyses can be reduced.

34 Although the formulation developed by Castelo et al. (2019) and Castelo et al. (in

35 press b) generate significant cost-savings and improve efficiency, they are limited to a

36 single soil model type, i.e. elastic-plastic (elastic-perfectly-plastic and non-linear elastic-

37 plastic, respectively). Therefore, further improvement is needed to capture the walking

38 behaviour with soils that develop a peak breakout resistance before reaching a plastic

39 plateau, as commonly seen in the operational areas mentioned above, and thus the

40 accuracy of pipeline walking results for analytical formulae is increased.

41 It has been found that there is a significant lack of knowledge about the influence

42 of pipe-soil interaction models on pipeline walking. Rong et al. (2009) acknowledge that

43 there is such an influence by stating: "The axial mobilization distances may have

44 significant effects on the axial walking. Unfortunately, limited literature is available 
45 about this topic." The latest joint industry project (JIP) research program, the SAFEBUCK

46 JIP (Bruton et al., 2007; Bruton and Carr, 2011a; Bruton and Carr, 2011b) has not

47 clarified what should be the treatment for axial pipe-soil interaction, when pipeline

48 walking is assessed. The SAFEBUCK JIP solely focused on the ideal rigid-plastic pipe-soil

49 interaction model.

50 In addition, the pipe-soil interaction standard, DNVGL-RP-F114 (DNVGL, 2017)

51 mentions: "In assessments of pipe walking, a low value of mobilisation distance creates a

52 higher rate of axial walking. To be conservative, a bi-linear fit to the non-linear response

53 should be a tangent fit to the initial part of the axial force-displacement response, which

54 represents the elastic recoverable part". Hence, no guidance has yet been given on how

55 to treat non-rigid-plastic soil models on pipeline walking assessments.

56 This paper investigates the impact on pipeline walking of a tri-linear soil

57 resistance model accounting for a peak break-out behaviour. It starts by a brief literature

58 review of the present methodology used to estimate the walking rate for elastic-plastic

59 soils (Castelo et al., 2019; Castelo et al., in press b). It then builds on the previous

60 knowledge to generate theoretical expressions for pipeline walking on peaky tri-linear

61 soils. Next, finite element analyses are performed to provide confirmation on the

62 theoretical framework. Finally, this paper generates a solution that allows an adjustment

63 for the original rigid-plastic analytical formulation (Bruton et al., 2010), so that the

64 requirement for finite element analyses can be avoided. 


\section{BACKGROUND TO PIPELINE WALKING}

\subsection{Downslope mechanism}

The seabed slope generates an asymmetry between the start-up (SUp) and

68 shutdown (SDown) phases in the effective axial force (EAF) profile for a fully mobilised

69 pipeline, as illustrated in Figure 1, where the rigid-plastic soil condition is considered.

70 This asymmetry causes the virtual anchor sections (VAS) to be separated by a given

71 distance, $X_{a b}$. For rigid-plastic soil representations, the virtual anchor sections

72 correspond to the maximum absolute effective axial force along the pipeline length, $L$.

73 Then, the distance $X_{a b}$ can be associated to the axial displacement, $\delta_{x}$, from a particular

74 load phase (start-up and shutdown phases), as presented by Figure 2. Because it tends to

75 create unbalanced displacements during different loading stages, the asymmetry in the

76 effective axial force profile is presently understood to be root cause of pipeline walking.

77 Accounting for more realistic soil conditions, the distance $X_{a b}$ cannot be

78 associated with maximum effective axial force. Therefore, $X_{a b}$ must be associated with

79 the stationary points (SP), as thoroughly explained in Castelo et al. (2019).

\section{$80 \quad 2.2 \quad$ Pipe-soil response}

81 Previous research on pipeline walking has treated soils as rigid-plastic (Carr et al.,

82 2003; Carr et al., 2006; Bruton et al., 2010) or as elastic-plastic (Castelo et al., 2019;

83 Castelo et al., in press b). However, it is known that some soils behave much more

84 complex, usually producing first a breakout peak resistance and then decreasing their

85 resistance to a residual plastic level. 
87 investigated peaky soils in general terms, none has gone through the specific impact on

88 pipeline walking. This paper focuses on a soil representation that accounts for breakout

89 soil resistance using a peaky tri-linear (3L) soil representation and how pipeline walking

90 may change due to this different soil condition.

\section{PROBLEM DEFINITION}

92 Downslope pipeline walking is dependent on three types of properties:

93 environmental, operational and those of the pipeline. This paper's parametric study uses

94 typical parameter ranges for these three properties.

95 The environmental parameters include seabed slope angle, $b$, and residual

96 friction coefficient, $\mu$, taken to be $2^{\circ}$ and 0.25 , respectively. The operational parameters

97 include temperature variation, $\Delta T$, and pipe submerged weight, $W$, assumed to be $100{ }^{\circ} \mathrm{C}$

98 and $0.4 \mathrm{kN} / \mathrm{m}$, respectively. The physical pipeline properties include steel outside

99 diameter, $O D$, steel wall thickness, $t$, and length, taken to be $0.3239 \mathrm{~m}, 0.0206 \mathrm{~m}$ and

$1005000 \mathrm{~m}$, respectively. Some additional environmental properties were taken as variables

101 for the parametric study, and they are related to the pipe-soil response (cases i - iv). The

102 full list of properties and parameters used in this study are provided in Table 1 and Table 1032.

$104 \quad$ Figure 3 presents a schematic axial force-displacement response for an ideal set

105 of peaky tri-linear soil cases. As investigated in White et al. (2011), it is known that

106 various aspects affect the cyclic behaviour of peaky soils. These aspects may be related, 107 but not limited, to the time interval between distinct movements, the varying pipeline 
108 embedment, etc. As a result, this paper takes into account two different extreme

109 conditions for the cyclic load phases:

110 • "EqualPeaks";

111 • "NoSUpPeak".

112 As the conditions' names suggest, the first condition, "EqualPeaks", behaves with

113 equal peaks for both loading and unloading phases - start-up and shutdown.

114 Alternatively, the second condition, "NoSUpPeak", behaves with no peak for start-up

115 phases, while peaky for shutdown phases. There is no clear understanding in the

116 literature of why the peak may exist for one load phase, while it may not occur for

117 another load phase, but this may be due to the differing waiting periods causing

118 different levels of consolidation or thixotropy (White et al. 2011). For example,

119 shutdowns are generally shorter duration than operating periods.

120 Consequently, the axial force-displacement responses, shown in Figure 3, need to

121 be updated to account for cyclic movements. Figure 4 shows the update to Figure 3,

122 presenting three hypothetical load phases for "EqualPeaks" and "NoSUpPeak"

123 conditions. The small numbered arrows indicate the loading path for each of the

124 conditions ("EqualPeaks" on top, and "NoSUpPeak" at the bottom of Figure 4).

125 According to previous experience (Castelo et al., 2019; Castelo et al., in press b),

126 the first load phase is not representative for the cyclic walking behaviour. At the initial

127 state, the nodes will displace a shorter distance to reach the relevant (either peak or

128 residual) force levels. Therefore, for simplicity, it is assumed that the first load phase 
129 does not peak; while for the cyclic load phases it will peak as prescribed by the

130 "EqualPeaks" or "NoSUpPeak" conditions.

131 In addition, although the authors acknowledge that intermediate peak cases may

132 occur in between "EqualPeaks" and "NoSUpPeak" conditions, these intermediate

133 conditions would be enveloped by these two extreme conditions. For this reason, the

134 intermediate cases are disregarded in this paper.

\section{ELASTIC-PERFECTLY-PLASTIC SOLUTION FOR PIPELINE WALKING}

136 From Castelo et al. (in press b) it is known that the walking rate for an elastic-

137 plastic pipe-soil response, $W R_{E P}$, can be obtained simply by subtracting twice the

138 equivalent mobilisation displacement, $\delta_{\text {mobEa }}$, from the walking rate for rigid-plastic soil,

$139 W R_{R P}$, as shown by equation (1):

$$
W R_{E P}=W R_{R P}-2 * \delta_{m o b E Q}
$$

140 where the walking rate for rigid-plastic soil can be estimated from Carr et al. (2006) and

141 the equivalent mobilisation displacement for a non-linear elastic-plastic pipe-soil

142 response can be obtained from Castelo et al. (in press b).

143 As another option, reorganizing equation (1), as also explained by Castelo et al.

144 (in press b), the walking rate for an elastic-plastic pipe-soil response, can be established

145 by multiplying the walking rate for rigid-plastic soil by a reduction factor based on the 146 equivalent mobilisation displacement and the non-walking mobilisation displacement, $147 \delta_{\text {null, }}$ as presented by equation (2):

$$
W R_{E P}=W R_{R P} *\left(1-\frac{\delta_{\text {mobEQ }}}{\delta_{\text {null }}}\right)
$$


148 where the non-walking mobilisation displacement, $\delta_{\text {null, }}$ can be achieved using equation

149 (3) - (Castelo et al., 2019):

$$
\delta_{\text {null }}=\frac{W R_{R P}}{2}
$$

As confirmed by Castelo et al. (in press b), the same reduction factor can be

151 applied to the distance between stationary points for an elastic-plastic pipe-soil

152 response, $X_{a b, E P}$, as presented below by equation (4):

$$
X_{a b, E P}=X_{a b, R P} *\left(1-\frac{\delta_{m o b E Q}}{\delta_{\text {null }}}\right)
$$

153 where the distance between stationary points for rigid-plastic soil, $X_{a b, R}$, can be

154 estimated from Bruton et al. (2010).

155 A parametric study has been performed using finite element analyses, to

156 investigate the peaky tri-linear pipe-soil responses seen in Figure 4, aiming on building

157 on equation (1) to create an accurate, simple and fast methodology to estimate pipeline

158 walking for this pipe-soil response type.

\section{FINITE ELEMENT METHODOLOGY}

160 The finite element model used in this paper is based on a straight pipeline laid on

161 a uniformly sloping seabed. The properties of this model are presented in Table 1 and

162 Table 2 (soil case ii). Table 2 also presents data used for the parametric study developed 163 later in this paper.

164 The $5000 \mathrm{~m}$ pipeline was represented by 5001 nodes connected by 5000 equal 165 Euler Bernoulli beam elements (B33 - 3 dimensional 3 noded elements in Abaqus 166 DASSAULT SYSTÈMES, 2014), creating a 1 metre "mesh" size. An overall sketch of the 167 finite element model used is presented by Figure 5. 
To represent the peaky tri-linear pipe-soil interaction, the soil was modelled as a

169 set of macro elements connected to each pipeline node, which were described as user

170 elements in user subroutine UEL coded in FORTRAN computer language.

\section{$171 \quad 5.1 \quad$ Peaky tri-linear pipe-soil interaction models}

172 Two different soil conditions were modelled for this paper: the "EqualPeaks" and

173 "NoSUpPeak" extreme conditions as shown in Figure 4.

174 For the "EqualPeaks" condition, the user element interface followed a constant

175 (positive) stiffness until a predefined peak elastic force, $F_{P}$, was attained. At this peak

176 force a constant (negative) stiffness was followed, so that the reaction force reduced up

177 to a residual plateau (residual plastic force, $F_{R}$ ). If the displacement was reversed, the

178 same behaviour could be observed for the spring-slider in the opposite direction.

179 For the "NoSUpPeak" condition, the user element interface applied the same

180 forces during loading as applied in the "EqualPeaks" condition. However, for start-up

181 phases, the forces did not present the peak, because once the reaction force achieved

182 the residual plateau, no further reaction was provided and the followed stiffness at this

183 point was zero, where the forces remained in the residual plateau.

\section{$184 \quad 5.2$ Loads}

185 In the analysis, the pipeline was heated up uniformly with temperature 186 increasing to $100{ }^{\circ} \mathrm{C}$. This value represents a combined equivalent effect of temperature 187 and pipe internal pressure (Hobbs, 1984).

188 The self-weight of the pipeline, $W$, and seabed slope angle, $b$, generate a sliding 189 component, $W_{\text {comp }}$, to the weight: 


$$
W_{\text {comp }}=W \sin \beta
$$

191 between the steady operational profile (start-up) and the rest condition (shutdown).

\subsection{Analysis description} were performed by running a sequence of load phases as follows:

$196 \quad$ 1. Applying boundary conditions and UEL properties;

2. Applying gravity to pipeline;

3. Applying temperature heating-up (start-up temperature);

4. Applying temperature cooling-down (shutdown temperature);

5. Iterating phases 3 and 4 (9 times);

6. Extracting results from simulations' outputs.

6 FINITE ELEMENT ANALYSIS RESULTS AND COMPARISON WITH RIGID-PLASTIC

\section{SOLUTION}

Figure 6 and Figure 7 show the effective axial force and the axial displacement 205 distribution, respectively, for the "EqualPeaks" condition applied to ideal case ii.

Figure 8 and Figure 9 show the effective axial force and the axial displacement

207 distribution for "NoSUpPeak" condition applied to ideal case ii. 
210 compared to the analytical calculations from Bruton et al. (2010), as shown in Table 4,

211 the deviation presented a remarkable margin.

212 The deviation between rigid-plastic calculations and finite element results (61 m

213 for the distance between stationary points and $0.066 \mathrm{~m} /$ cycle for the walking rate) is

214 justified by the fact that the finite element analyses considered a more realistic soil.

215 Instead of using a basic soil approximation, rigid-plastic, the analyses considered a more

216 realistic soil response, peaky tri-linear pipe-soil interaction.

217 To estimate the realistic results for the distance between stationary points and

218 for the walking rate, a closed-form solution is outlined for the peaky tri-linear pipe-soil

219 response, as was done in Castelo et al. (2019) and Castelo et al. (in press b).

2207 REVISED CLOSED-FORM SOLUTION FOR THE DISTANCE BETWEEN STATIONARY $221 \quad$ POINTS FOR PEAKY TRI-LINEAR SOILS $-\boldsymbol{X}_{a b, 3 L}$

222 From Castelo et al. (in press b) where the soil is treated as a non-linear elastic-

223 plastic spring, it is known that the distance between stationary points, $X_{a b, E P}$, is equal to

224 the distance between stationary points for rigid-plastic soils, $X_{a b, R P}$, multiplied by a 225 reduction factor, which is based on the equivalent mobilisation displacement, $\delta_{\text {mobEQ }}$,

226 and the non-walking mobilisation displacement, $\delta_{\text {null, }}$ as shown by equation (4).

227 Alternatively, for a peaky tri-linear pipe-soil behaviour, the equivalent 228 mobilisation displacement, $\delta_{\text {mobEQ }}$, might be substituted by an ideal mobilisation 229 displacement, $\delta_{m o b}$.

$$
X_{a b, 3 L}=X_{a b, R P} *\left(1-\frac{\delta_{m o b}{ }^{\prime}}{\delta_{\text {null }}}\right)
$$


Journal of Offshore Mechanics and Arctic Engineering

2308 REVISED ANALYTICAL SOLUTION FOR THE WALKING RATE FOR PEAKY TRI-LINEAR

$231 \quad$ SOILS $-W R_{3 L}$

232 From Castelo et al. (in press b), which treated the soil as a non-linear elastic-

233 plastic spring, it is known that the walking rate, $W R_{3 L}$, is equal to the walking rate for

234 rigid-plastic soils, $W R_{R P}$, multiplied by a reduction factor based on the equivalent

235 mobilisation displacement, $\delta_{\text {mobEQ }}$, and the non-walking mobilisation displacement, $\delta_{\text {null, }}$

236 as previously shown by equation (2).

237 Analogously to $X_{a b, 3 L}$, for a peaky tri-linear pipe-soil behaviour, the equivalent 238 mobilisation displacement, $\delta_{\text {mobEQ }}$, might be substituted by an ideal mobilisation 239 displacement, $\delta_{m o b}$.

$$
W R_{3 L}=W R_{R P} *\left(1-\frac{\delta_{m o b}{ }^{\prime}}{\delta_{\text {null }}}\right)
$$

241 As firstly developed by Castelo et al. (2019) and further expanded by Castelo et

242 al. (in press b) for the elastic correction, the tri-linear correction, Corr 3 , for the walking

243 rate predictions can be obtained by doubling the division of the unload-reload area,

$244 A_{\text {Unload-Reload, }}$ by the variation of residual plastic force, $\Delta F_{R}$. However, differently to elastic-

245 plastic soils, peaky tri-linear pipe-soil interactions have an additional area, created by the 246 peak resistance, but the influence of the peak resistance is so small, that this additional 247 area can be safely ignored resulting in:

$$
\operatorname{Corr}_{3 L}=2\left(\frac{A_{\text {Unload-Reload }}}{\Delta F_{R}}\right)
$$


249 can be described with a similar procedure from Castelo et al. (2019) and Castelo et al. (in 250 press b), as outlined by equation (9):

$$
\delta_{m o b}{ }^{\prime}=\frac{\operatorname{Corr}_{3 L}}{2}=\left(\frac{A_{\text {Unload-Reload }}}{\Delta F_{R}}\right)
$$

As another option, since the soil behaves linearly, $\delta_{\text {mob }}$ can also be written as:

$$
\delta_{m o b}{ }^{\prime}=\frac{F_{R} * \delta_{m o b P}}{F_{P}}
$$

252 where $F_{R}$ is the residual plastic force, $F_{P}$ is the peak elastic force, and $\delta_{m o b P}$ is the

253 mobilisation displacement where the peak elastic force is achieved.

254 Now, using the values provided in Table $2, \delta_{\text {mob }}$ was calculated for cases i - iv to

255 be $0.065,0.032,0.043$ and $0.052 \mathrm{~m}$, respectively; while, equations (6) and (7) were

256 rewritten, accounting for equation (10), as:

$$
\begin{gathered}
X_{a b, 3 L}=X_{a b, R P}\left(1-\frac{F_{R}}{F_{P}} \frac{\delta_{m o b P}}{\delta_{\text {null }}}\right) \\
W R_{3 L}=W R_{R P} *\left(1-\frac{F_{R}}{F_{P}} \frac{\delta_{\text {mobP }}}{\delta_{\text {null }}}\right)
\end{gathered}
$$

257 Hence, using equations (11) and (12) in association to the values provided by

258 Table 1, Table 2 and Table 4, the distance between stationary points and the walking 259 rate were obtained, as presented by Table $5^{*}$.

10 FINITE ELEMENT ANALYSES PARAMETRIC STUDY FOR PEAKY TRI-LINEAR PIPE-SOIL

\section{INTERACTION}

262

The following parametric study validates the above solutions for the distance between stationary points' and walking rate for peaky tri-linear soils.

\footnotetext{
* The authors understand that $1 \mathrm{~m}$ lies inside the acceptable deviation given that this is the mesh spacing.
} 
265 previously explained in section 3. For simplicity, pipeline length, pipeline submerged 266 operational weight (accounting for content), residual friction coefficient and the overall

267 route slope were kept constant, although the soil resistance was varied, as shown, for 268 the ideal cases i - iv, in Table 2, Figure 3 and Figure 4.

\section{$269 \quad 10.1$ Ideal mobilisation displacement $-\boldsymbol{\delta}_{\text {mob }}{ }^{\prime}$}

270 Each of the parametric study cases tested had their own ideal mobilisation

271 displacement, $\delta_{m o b^{\prime}}$, value according to equation (10) as shown in section 9.

$272 \quad$ Figure 10 presents the tri-linear correction results from the numerical solutions

273 (finite element models) plotted against the values calculated using equation (8). The

274 "EqualPeaks" and the "NoSUpPeak" soil conditions are represented by square and

275 circular markers, respectively. The triangles represent elastic-perfectly-plastic conditions,

276 accounting for the ideal mobilisation displacement - these were used to prove the

277 applicability of the ideal mobilisation displacement methodology. Cases i, ii, iii and iv are 278 indicated in the figure.

279 Figure 10 shows a very strong agreement between the tri-linear correction

280 obtained from the finite element analysis and the results calculated using the proposed 281 equation.

282 For elastic-plastic soil conditions, when the equivalent mobilisation displacement,

$283 \delta_{\text {mobEQ }}$, nears the value of the non-walking mobilisation displacement, $\delta_{\text {null, }}$ the walking 284 rate tends to diminish up to zero and the walking phenomenon ceases (Castelo et al., 285 2019; Castelo et al., in press b). Analogously, to peaky tri-linear soils, when the ideal 
Journal of Offshore Mechanics and Arctic Engineering

286 mobilisation displacement, $\delta_{m o b^{\prime}}$, nears $\delta_{\text {null }}$ the walking rate also tends to diminish up to

287 zero and the walking phenomenon ceases.

28810.2 Distance between stationary points for peaky tri-linear soil $-X_{a b, 3 L}$

289 Equation (10) is applicable to finding the ideal mobilisation displacement.

290 Consequently, equation (11) must be applicable to finding the distance between the

291 stationary points. To confirm, the finite element model outputs were compared with the

292 calculated values from equation (11).

293 Figure 11 presents the results for the distance between stationary points using

294 numerical solutions (finite element models) plotted against the values calculated using

295 equation (11). The "EqualPeaks" and the "NoSUpPeak" soil conditions are represented

296 by square and circular markers, respectively. The triangles represent elastic-perfectly-

297 plastic conditions, accounting for the ideal mobilisation displacement. Cases i, ii, iii and iv 298 are indicated in the figure.

299 Figure 11 shows a very strong agreement for the distance between stationary 300 points obtained from the finite element analysis and the results calculated using the 301 proposed equation.

$302 \quad 10.3$ Walking rate for peaky tri-linear soil $-W R_{3 L}$

$303 \quad$ Figure 12 presents the walking rate results from the numerical solutions (finite 304 element models) plotted against the values calculated using equation (12). The 305 "EqualPeaks" and the "NoSUpPeak" soil conditions are represented by square and 306 circular markers, respectively. The triangles represent elastic-perfectly-plastic conditions, 
307 accounting for the ideal mobilisation displacement. Cases i, ii, iii and iv are indicated in 308 the figure.

Figure 12 shows a very strong agreement between the walking rates obtained

310 from the finite element analysis and the results calculated using the proposed equation.

311 Overall, the results show that equation (12) - as presented by Table 6 - gives a

312 true representation of the effects of peaky tri-linear soil springs on pipeline walking.

313 Finally, equation (1) can be translated for peaky tri-linear soils as equation (13):

$$
W R_{3 L}=W R_{R P}-2 \delta_{m o b}{ }^{\prime}
$$

315 by subtracting twice the ideal mobilisation displacement, $\delta_{m o b}$, from the walking rate for

316 rigid-plastic soils, $W R_{R P}$. force values and ultimately change the $\Delta S_{S}$ expression.

While obtaining the effective axial force variation over the distance between

326 stationary points is important, previous experience (Castelo et al., 2019; Castelo et al., in

327 press b), shows that $\Delta S_{s}$ revision will not have a significant impact on finding the walking 328 rate for peaky tri-linear soils. Furthermore, confidence in the numerical solutions 
329 obtained in previous research, and the use of similar approaches (Castelo et al., 2019;

330 Castelo et al., in press b), suggest that the numerical results will be sufficient to prove

331 the applicability of equations (10), (11), (12) and (13).

33212 LIMITATIONS \& FUTURE WORK

333 The emerging methodology has not been tested for implications on lateral

334 buckling (also referred to as Euler buckling). It has been tested in previous stages of

335 research for various pipeline lengths, which has been proved to have no influence over

336 the overall findings (Castelo et al., 2019; Castelo et al., in press b). For variable slopes,

337 the authors foresee further research to be published in the near future (Castelo et al., in

338 press a).

339 In general terms, there are no limitations for the applicability of this

340 methodology as long as the pipeline route respect a uniformly sloped seabed with no

341 lateral buckles.

\section{CONCLUSIONS \& FINAL REMARK}

343 This paper provides a strategy to solve downslope pipeline walking problems

344 considering peaky tri-linear soils. Different properties of tri-linearity (within the peaky

345 soil model type) have been considered, leading to an innovative analytical solution.

346 The innovation is summarised as the multiplying factor introduced in equation

347 (12) and highlighted below:

$$
\left(1-\frac{F_{R}}{F_{P}} \frac{\delta_{m o b P}}{\delta_{\text {null }}}\right)
$$


350 adjust the original rigid-plastic solution in a way to make the walking assessment results

351 more accurate.

352 The new solution, based on the factor highlighted in equation (14), was

353 benchmarked and validated against a set of finite element analyses.

354 Currently applied analytical solutions do not consider the soil model type with a

355 peak, and it is known that they can provide inaccurate walking patterns.

356 Therefore, this paper resolves how the fundamental closed-form solution for

357 rigid-plastic soils must be adjusted to allow for peaky tri-linear soils reducing the

358 requirement for numerical modelling which can be time- and resource-consuming in

359 early stages of design activities, such as preliminary estimates for downslope pipeline 360 walking.

\section{ACKNOWLEDGMENT}

363 This research forms part of the activities of the Centre for Offshore Foundation 364 Systems (COFS). The authors acknowledge support from The University of Western 365 Australia, Shell, and the Australian Research Council. 
Journal of Offshore Mechanics and Arctic Engineering

367 NOMENCLATURE

3L Tri-Linear

E Steel Young's Modulus

EAF effective axial force

Fp peak elastic force

$F_{R} \quad$ residual plastic force

JIP joint industry project

$L \quad$ pipeline length

$O D \quad$ steel outside diameter

RP rigid-plastic

SDown shutdown phase

SUp start-up phase

SP stationary point

$t \quad$ steel wall thickness

VAS virtual anchor section

W pipeline submerged weight

$W_{\text {comp }} \quad$ pipeline weight component

WR walking rate

$x \quad$ axial coordinate along pipe length 


\begin{tabular}{|c|c|}
\hline$X_{a b}$ & distance between stationary points \\
\hline$\alpha$ & steel thermal expansion coefficient \\
\hline B & seabed slope angle \\
\hline$\Delta S_{S}$ & $\begin{array}{l}\text { effective axial force variation over the distance between stationary } \\
\text { points }\end{array}$ \\
\hline$\Delta T$ & temperature variation \\
\hline$\delta_{m o b}{ }^{\prime}$ & ideal mobilisation distance \\
\hline$\delta_{m o b P}$ & peak elastic force mobilisation displacement \\
\hline$\delta_{m o b R}$ & residual plastic force mobilisation displacement \\
\hline$\delta_{\text {null }}$ & non-walking mobilisation distance \\
\hline$\delta_{x}$ & axial displacement \\
\hline$\mu$ & residual friction coefficient \\
\hline$v$ & steel Poisson coefficient \\
\hline
\end{tabular}




\section{REFERENCES}

370

371

372

373

374

375

376

377

378

379

380

381

382

383

384

385

386

387

388

389

390

391

392

393

394

395

396

397

398

399

400

401

402

403

404

405

406

407

408

409
Bruton, D. and Carr, M. (2011a) 'Lateral Buckling and Pipeline Walking - Overview of the SAFEBUCK JIP', Proceedings of the Subsea Australasia Conference 2011.

Bruton, D. and Carr, M. (2011b) 'Overview of the SAFEBUCK JIP', Proceedings of the Offshore Pipeline Technology Conference. doi: 10.4043/21671-MS.

Bruton, D., Carr, M. and White, D. (2007) 'The Influence of Pipe-Soil Interaction on Lateral Buckling and Walking of Pipelines - the SAFEBUCK JIP', Proceedings of the International Conference on Offshore Site Investigation and Geotechnics.

Bruton, D., Sinclair, F. and Carr, M. (2010) 'Lessons Learned from Observing Walking of Pipelines with Lateral Buckles, Including New Driving Mechanisms and Updated Analysis Models', Proceedings of the Offshore Technology Conference. doi: 10.4043/20750-MS.

Carr, M., Bruton, D. and Leslie, D. (2003) 'Lateral Buckling and Pipeline Walking, a Challenge for Hot Pipelines', Proceedings of the Offshore Pipeline Technology Conference.

Carr, M., Sinclair, F. and Bruton, D. (2006) 'Pipeline Walking - Understanding the Field Layout Challenges and Analytical Solutions Developed for the Safebuck JIP', Proceedings of the Offshore Technology Conference. doi: 10.2118/120022-PA.

Castelo, A., White, D. and Tian, Y. (2019) 'Simple Solutions for Downslope Pipeline Walking on Elastic-Perfectly-Plastic Soils', Ocean Engineering. Elsevier Ltd, 172, pp. 671683. doi: 10.1016/j.oceaneng.2018.11.037.

Castelo, A., White, D. and Tian, Y. (in press a) 'Gravity Driven Pipeline Walking on Variable Slopes', International Journal of Offshore and Polar Engineering.

Castelo, A., White, D. and Tian, Y. (in press b) 'Solving Downslope Pipeline Walking on Non-Linear Elastic-Plastic Soils', Marine Structures.

DASSAULT SYSTÈMES (2014) 'Abaqus 6.14 Analysis User's Manual'. Providence, RI, USA: Dassault Systèmes Simulia Corporation.

DNVGL (2017) DNVGL-RP-F114: Pipe-Soil Interaction for Submarine Pipelines.

Hobbs, R. (1984) 'In-Service Buckling of Heated Pipelines', Journal of Transportation Engineering, 110(2), pp. 175-189. doi: 10.1061/(ASCE)0733-947X(1984)110:2(175).

Rong, H., Inglis, R., Bell, G., Huang, Z., and Chan, R. (2009) 'Evaluation and Mitigation of Axial Walking with a Focus on Deep Water Flowlines', Proceedings of the Offshore Technology Conference. doi:10.4043/19862-MS

Tornes, K., Ose, B., Jury, J., Thomson, P. et al. (2000) 'Axial Creeping of High Temperature Flowlines Caused by Soil Ratcheting', Proceedings of the ETCE/ OMAE Joint Conference, Energy for the New Millennium. Vol. 2, pp. 1229-1240.

White, D., Ganesan, S., Bolton, M., Bruton, D., Ballard, J-C., Langford, T. et al. (2011) 'SAFEBUCK JIP - Observations of Axial Pipe-Soil Interaction from Testing on Soft Natural Clays', Proceedings of the Offshore Technology Conference. doi: 10.4043/21249-MS. 
Journal of Offshore Mechanics and Arctic Engineering

Figure 1 Effective axial force diagrams for start-up and shutdown phases

Figure 2 Axial displacement diagrams for start-up and shutdown phases

Figure $3 \quad$ Tri-linear soil responses

Figure $4 \quad$ Tri-linear soil responses for cyclic movements

Figure 5 Effective axial force for tri-linear strategy case ii - EqualPeaks (Zoom) Finite element model sketch

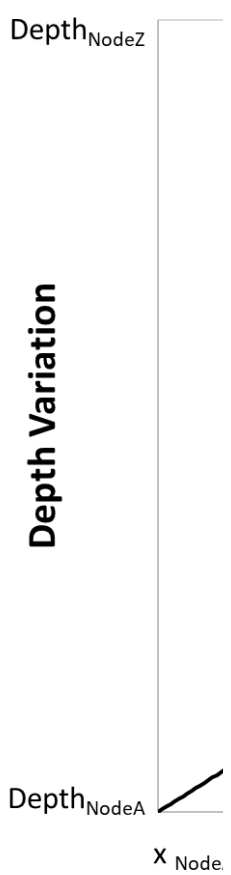

Figure 6

Figure 7 Axial displacement for tri-linear strategy case ii - EqualPeaks (Zoom)

Figure 8 Effective axial force for tri-linear strategy case ii - NoSUpPeak (Zoom) 
Journal of Offshore Mechanics and Arctic Engineering

Figure 9 Axial displacement for tri-linear strategy case ii- NoSUpPeak (Zoom)

Figure 10 Tri-linear correction results

Figure 11 Distance between stationary points results

Figure 12 Walking rate results

411 
Journal of Offshore Mechanics and Arctic Engineering

\section{Table Caption List}

Table $1 \quad$ General properties

Table $2 \quad$ Case properties

Table 3 Tri-linear finite element analysis results for soil case ii

Table $4 \quad$ Rigid-plastic calculation results

Table $5 \quad$ Analytical results

Table $6 \quad$ Tri-linear finite element analyses results 


\section{FIGURES}

415 Figure 1 Effective axial force diagrams for start-up and shutdown phases

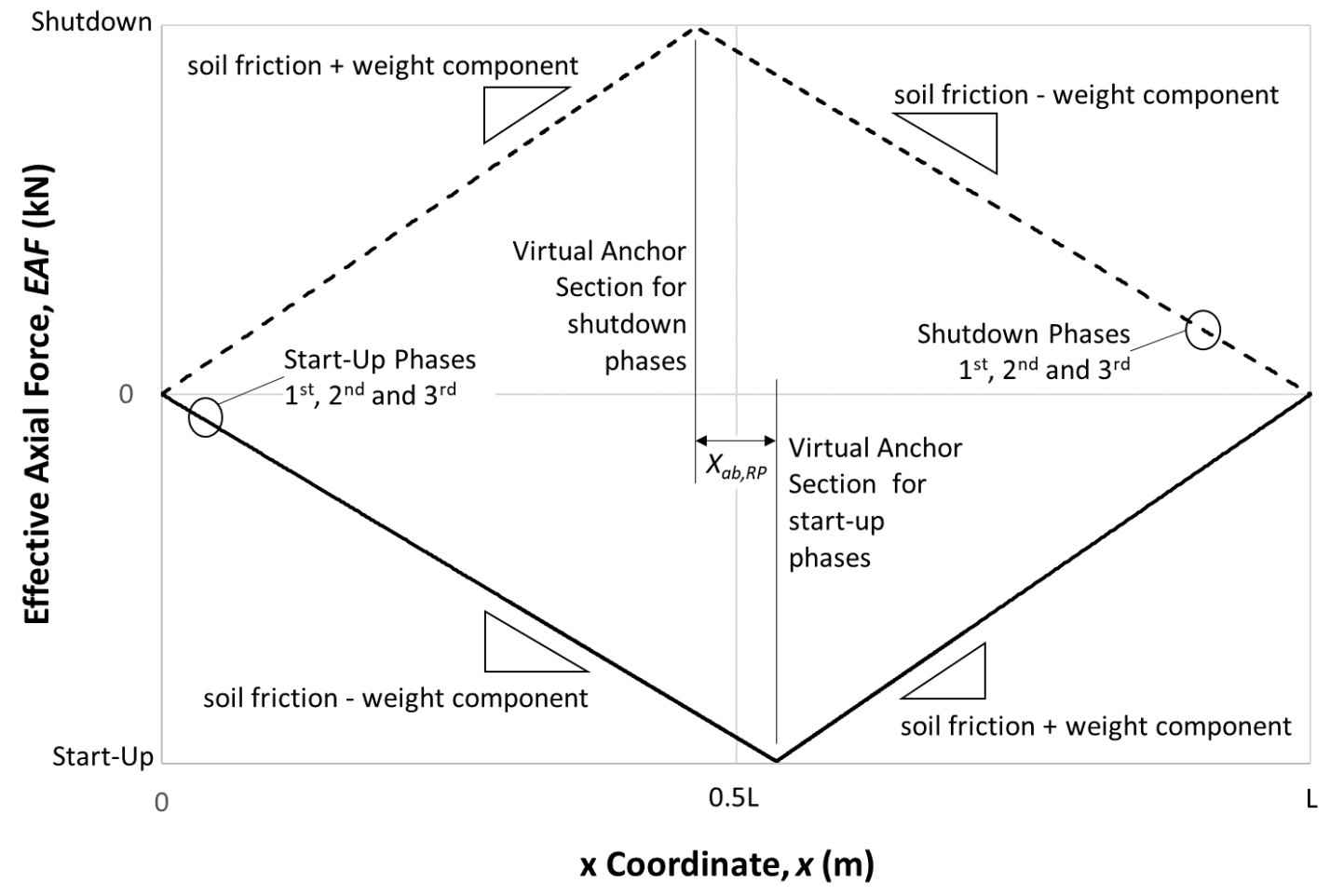


Figure 2 Axial displacement diagrams for start-up and shutdown phases

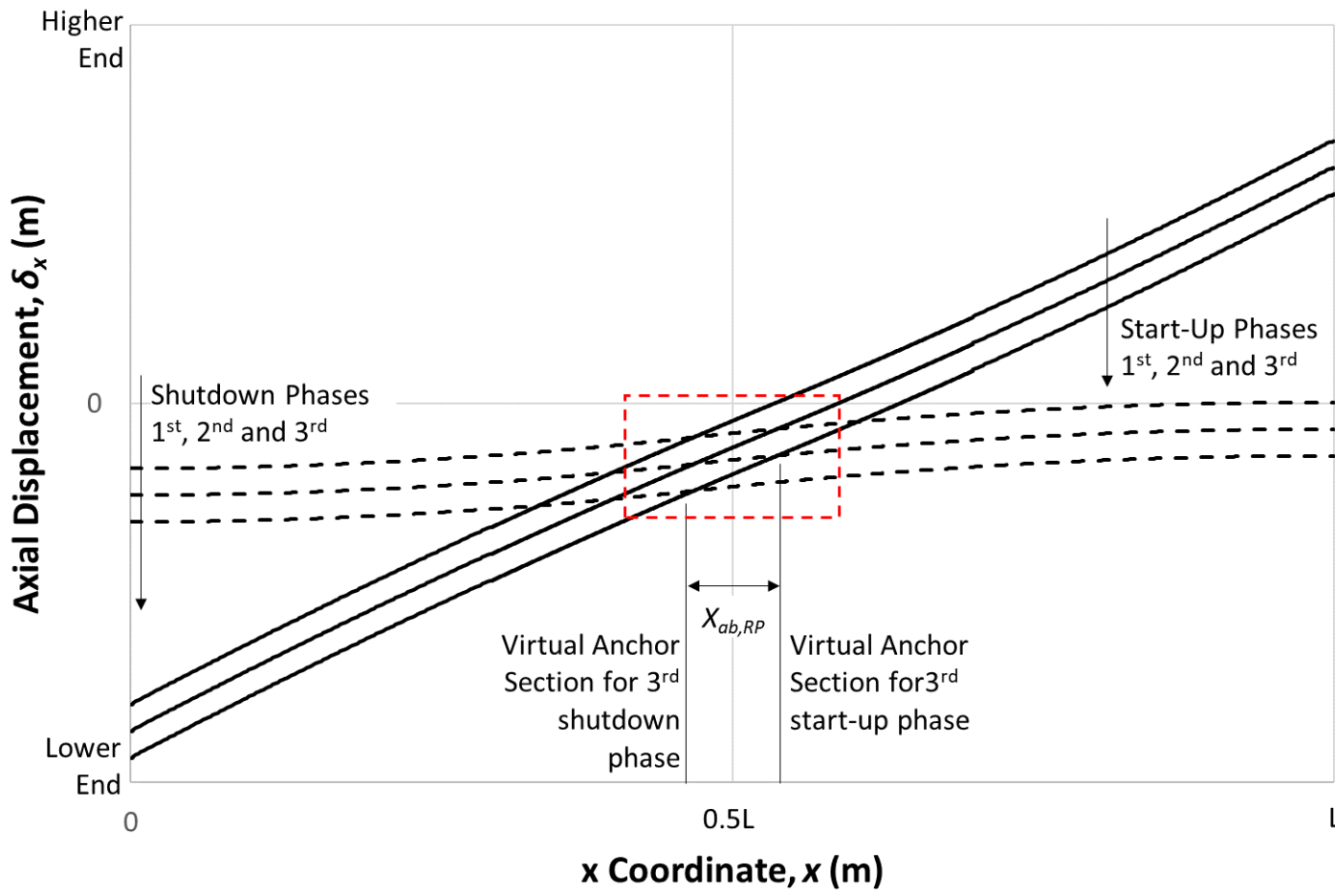

420

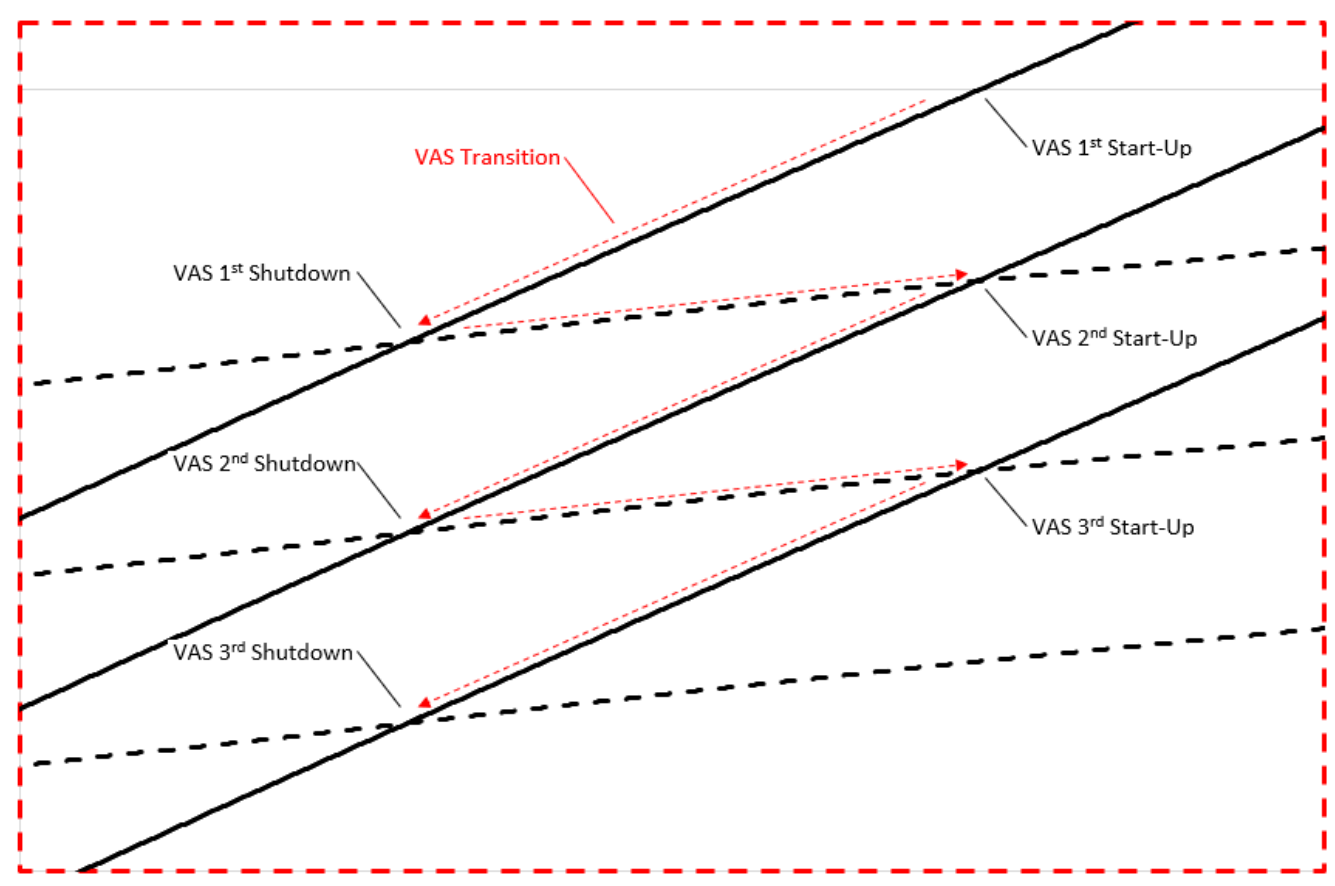


Journal of Offshore Mechanics and Arctic Engineering

\section{Figure 3 Tri-linear soil responses}

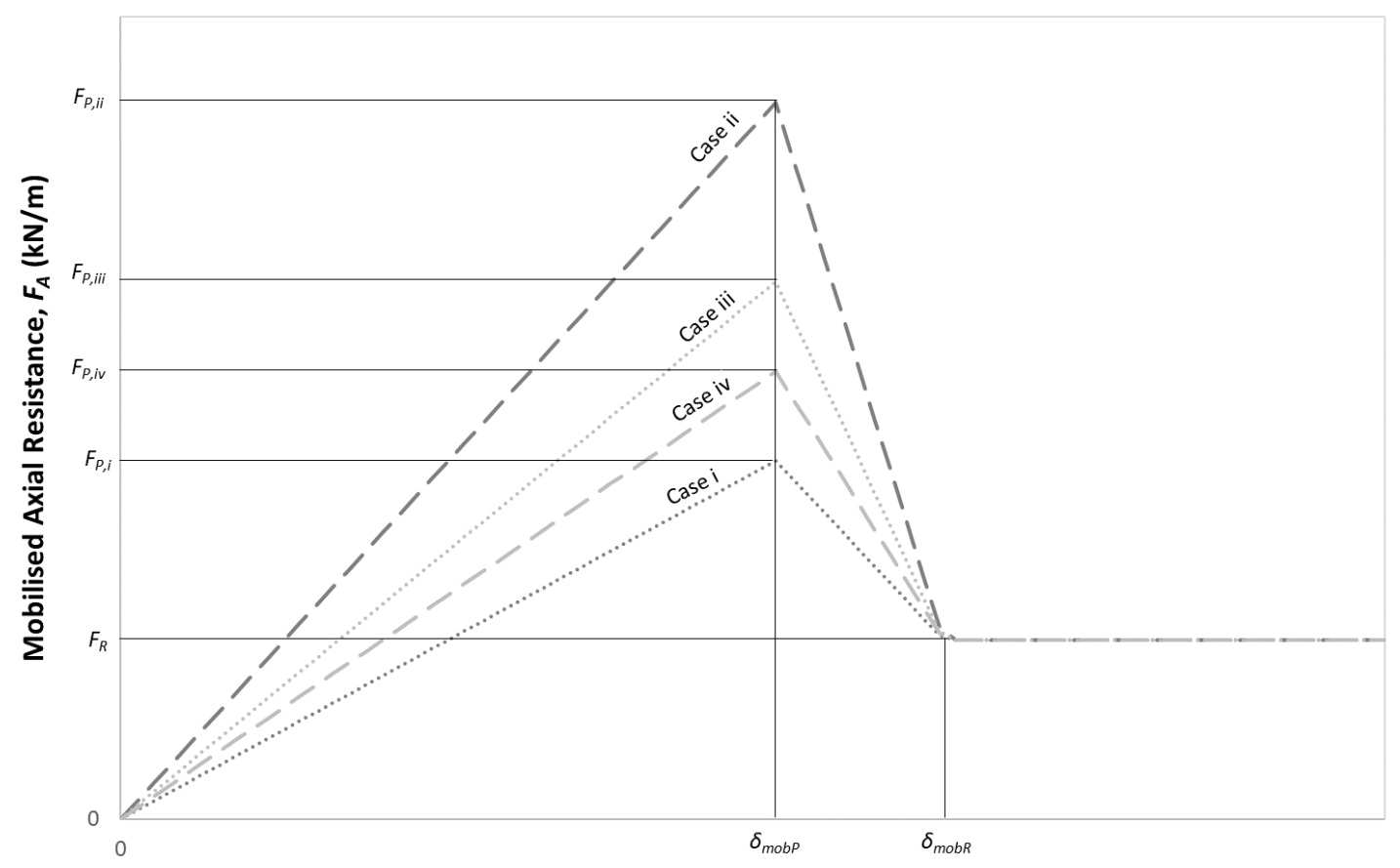

425

Axial Displacement, $\delta_{x}(\mathrm{~m})$

426

427 


\section{8}

\section{Figure 4 Tri-linear soil responses for cyclic movements}

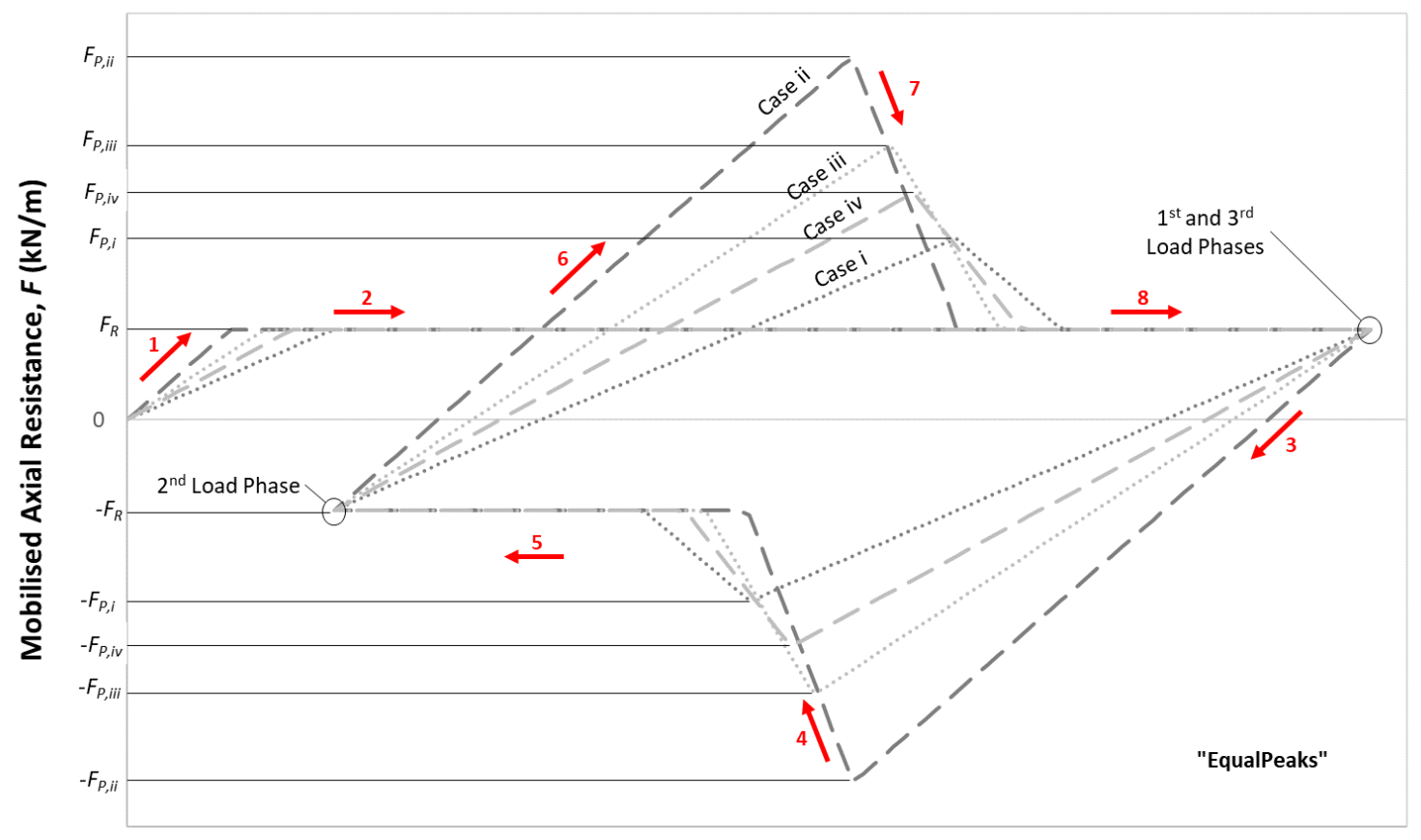

0

429

Axial Displacement, $\delta_{x}(\mathrm{~m})$

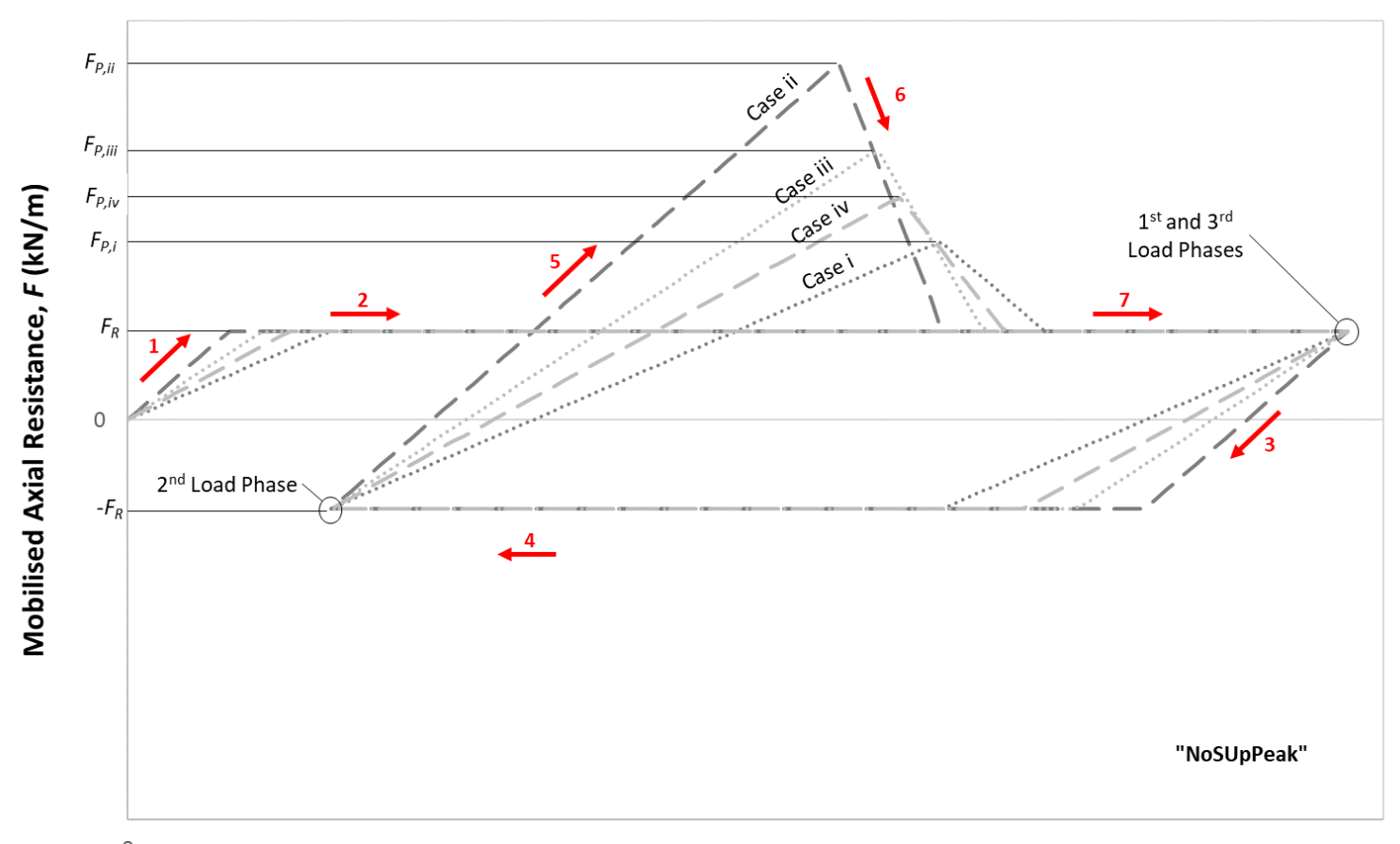

Axial Displacement, $\delta_{x}(\mathrm{~m})$

430

431

432 
Journal of Offshore Mechanics and Arctic Engineering

433 Figure 5 Finite element model sketch

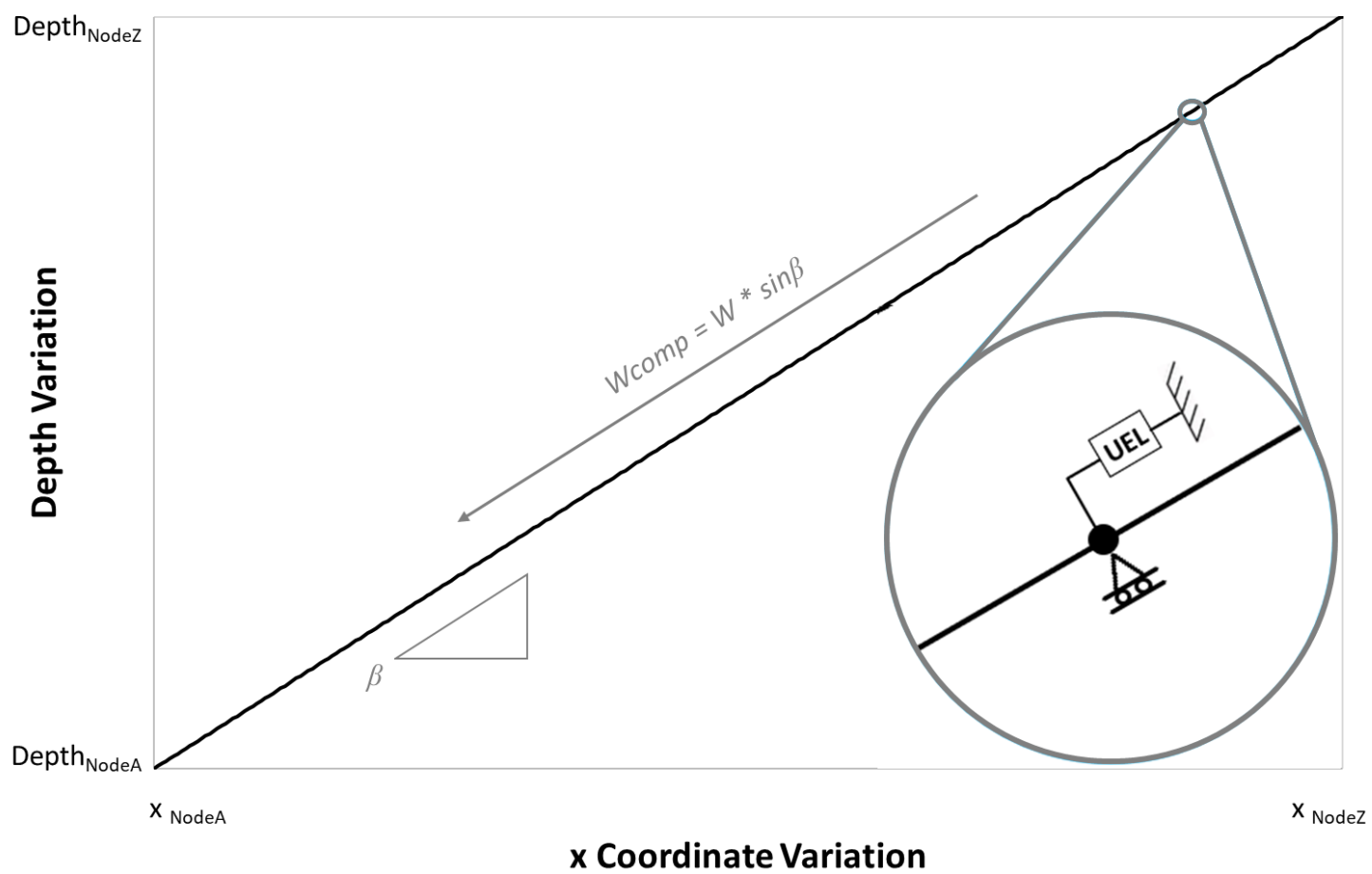

434

435

436 
Figure 6 Effective axial force for tri-linear strategy case ii - EqualPeaks (Zoom)

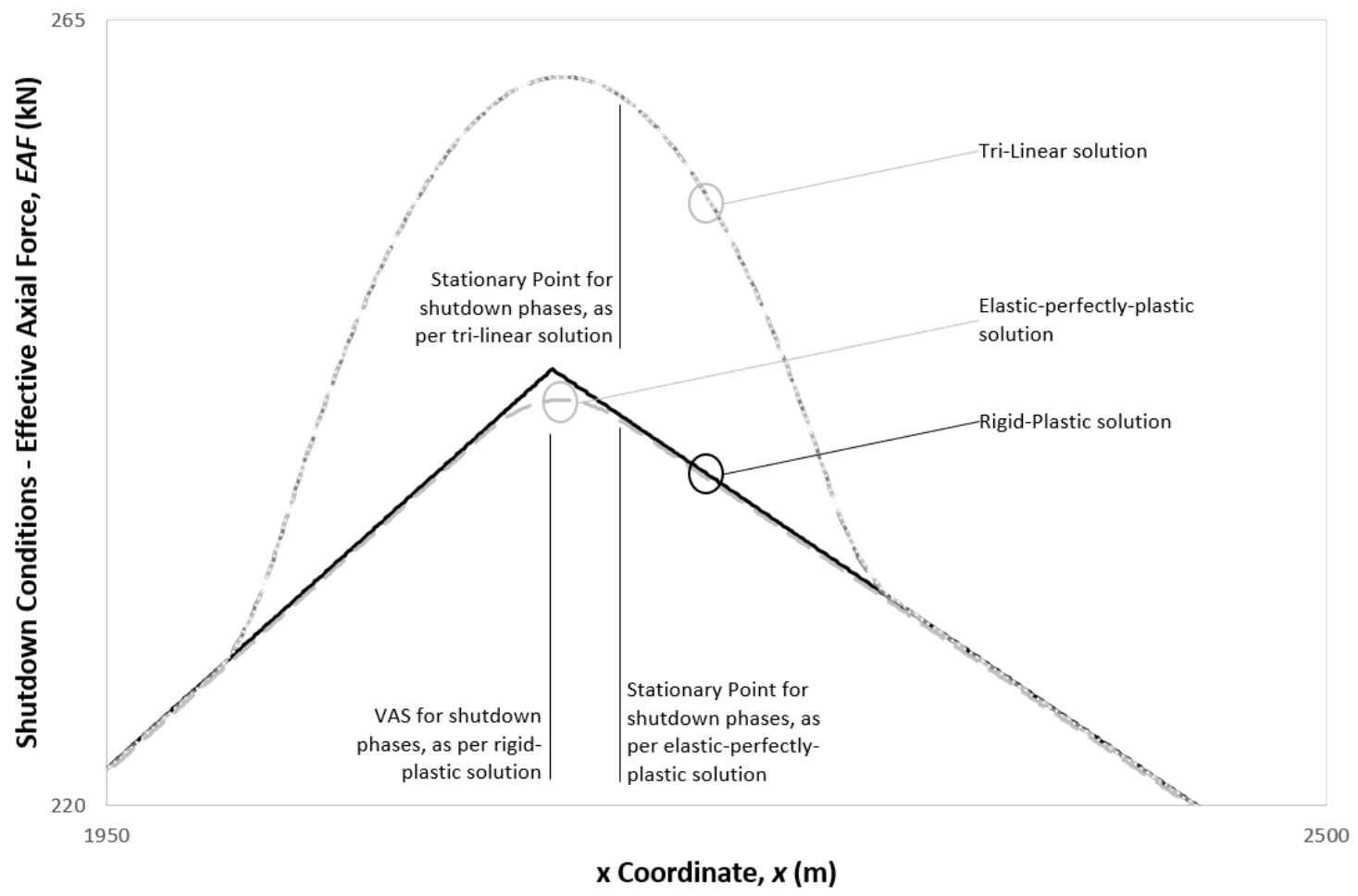

438

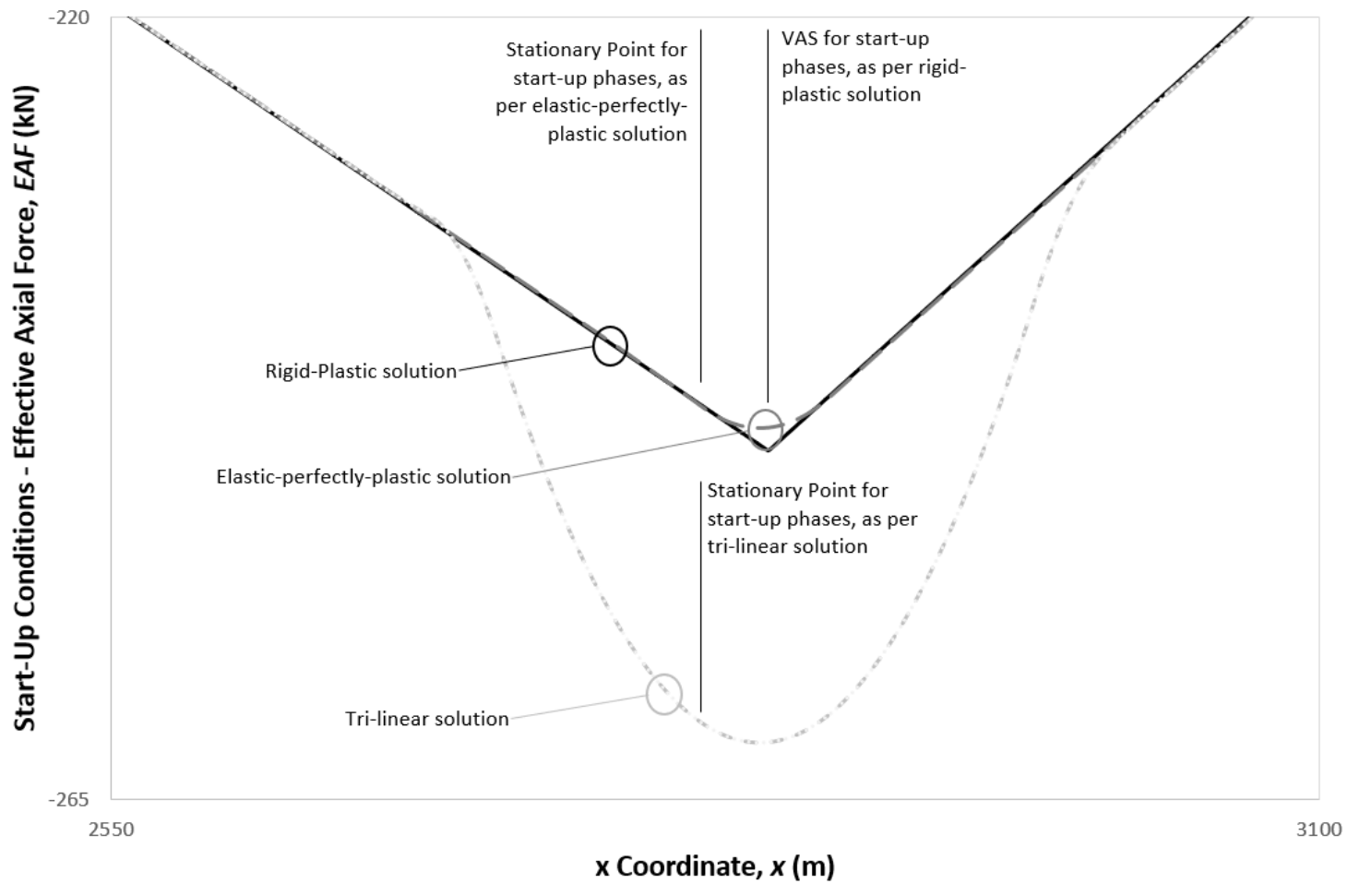




\section{Figure 7 Axial displacement for tri-linear strategy case ii - EqualPeaks (Zoom)}

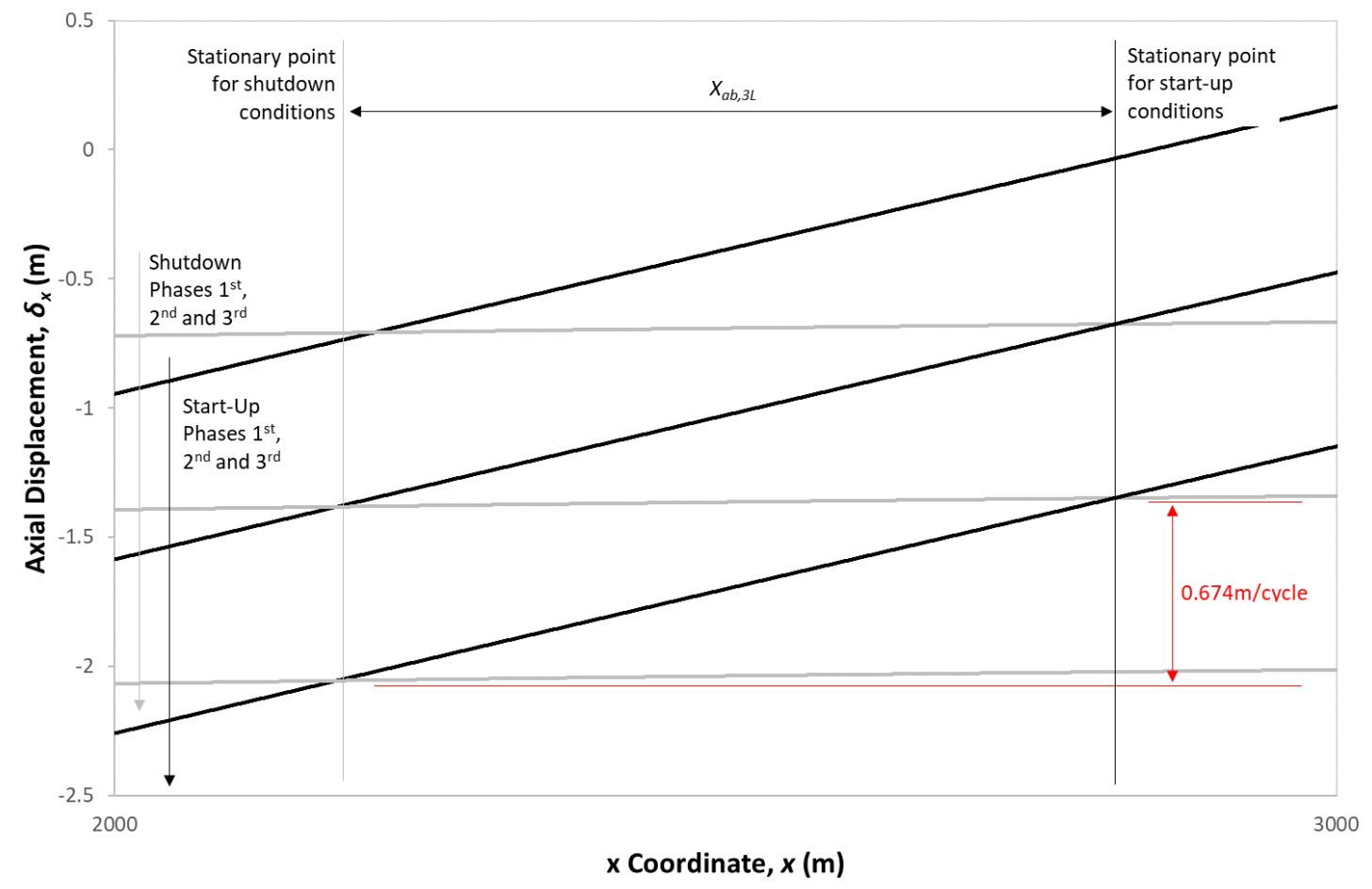

443

444

445 


\section{Figure 8 Effective axial force for tri-linear strategy case ii - NoSUpPeak (Zoom)}

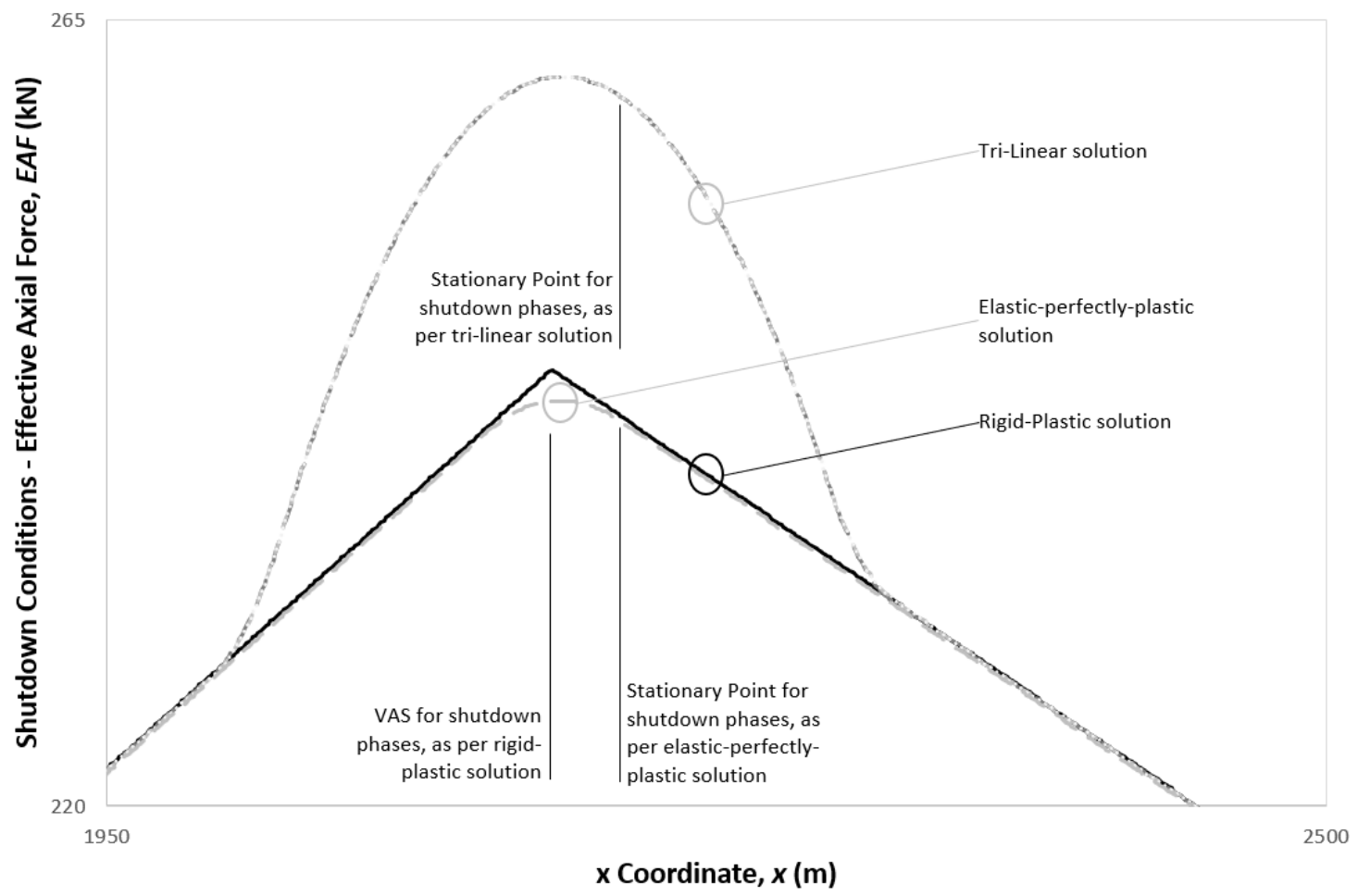

447

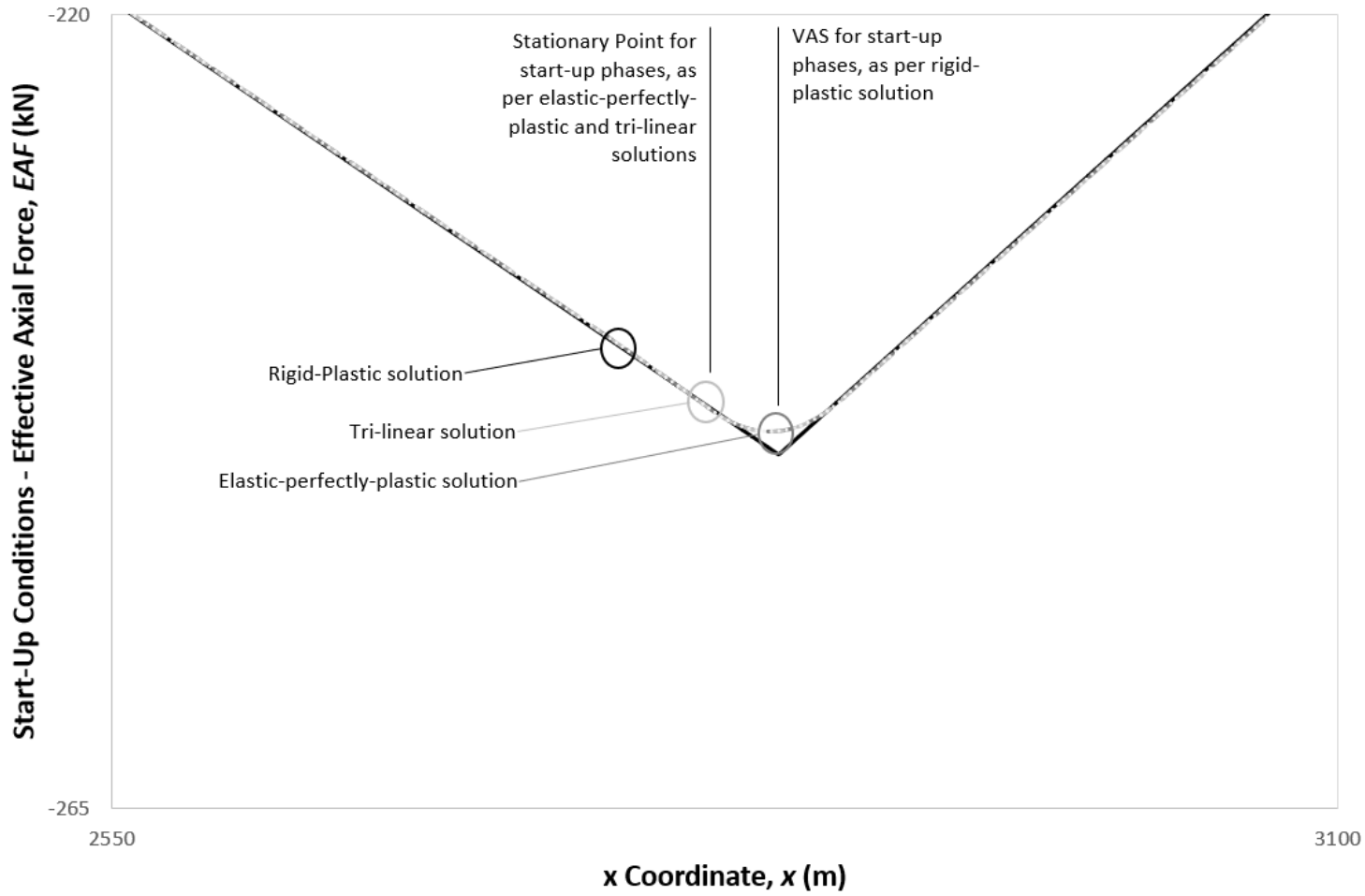


451 Figure 9 Axial displacement for tri-linear strategy case ii- NoSUpPeak (Zoom)

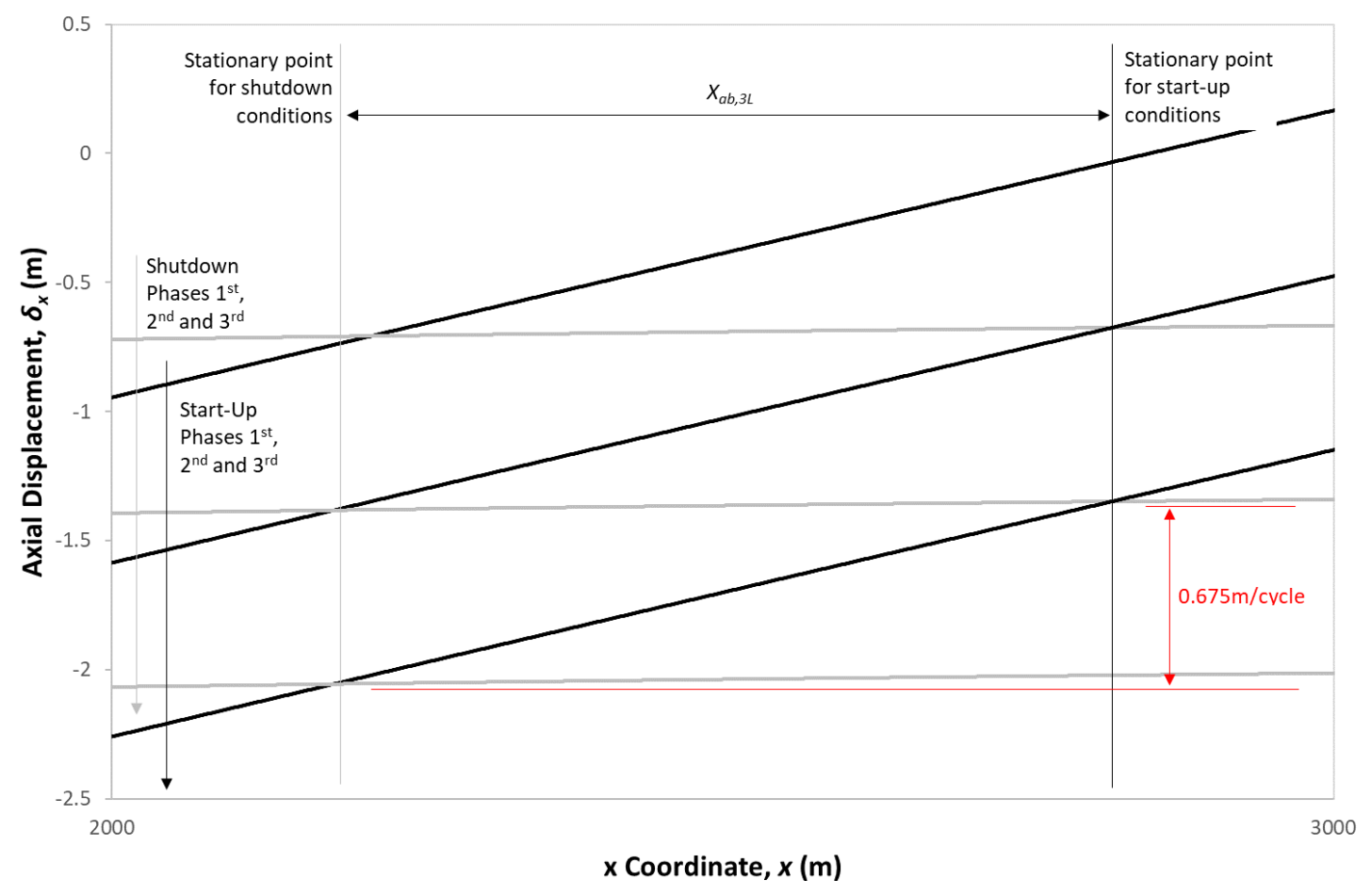

452

453

454 
Journal of Offshore Mechanics and Arctic Engineering

\section{Figure 10 Tri-linear correction results}

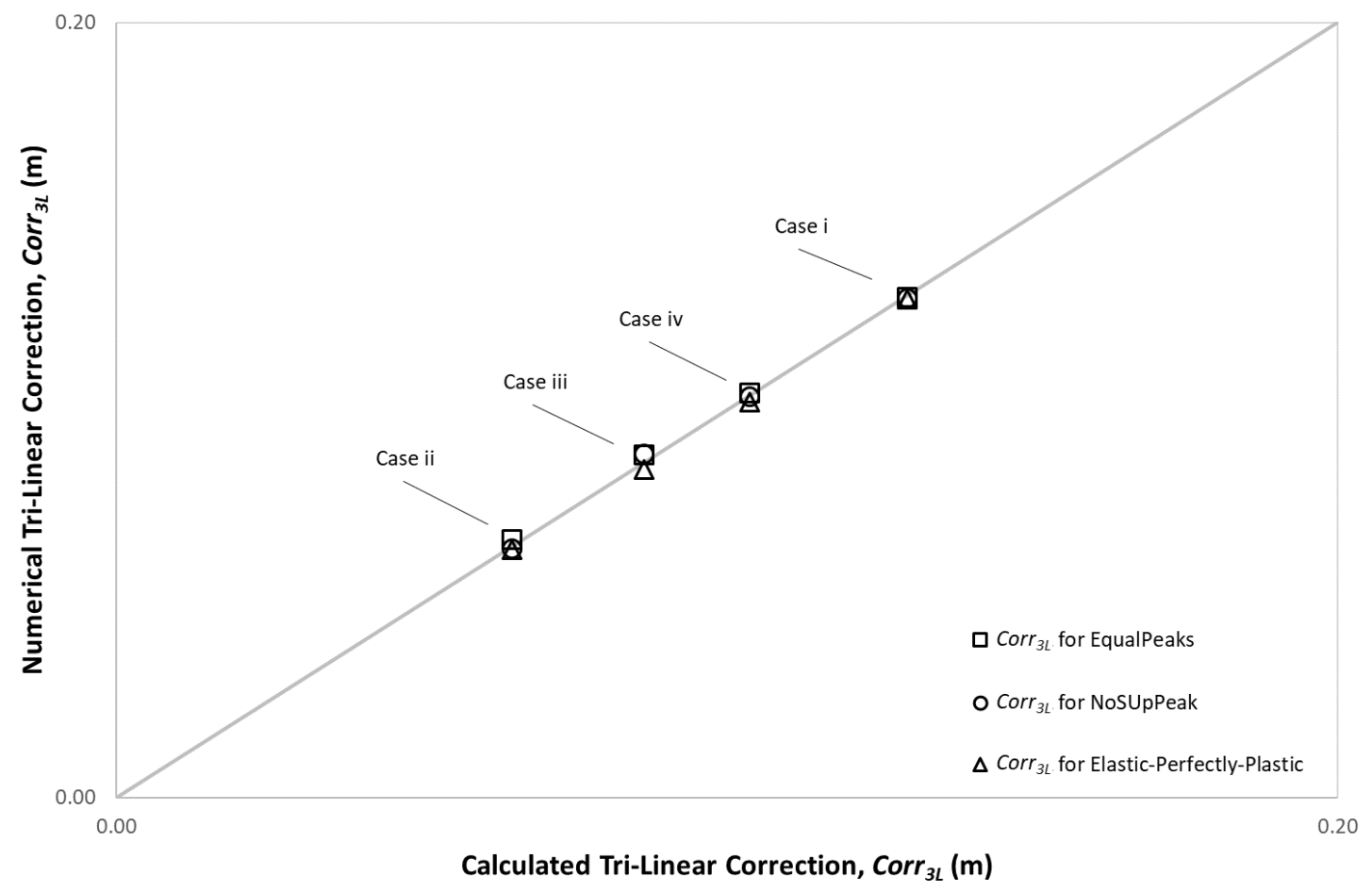

456

457 
Journal of Offshore Mechanics and Arctic Engineering

\section{Figure 11 Distance between stationary points results}

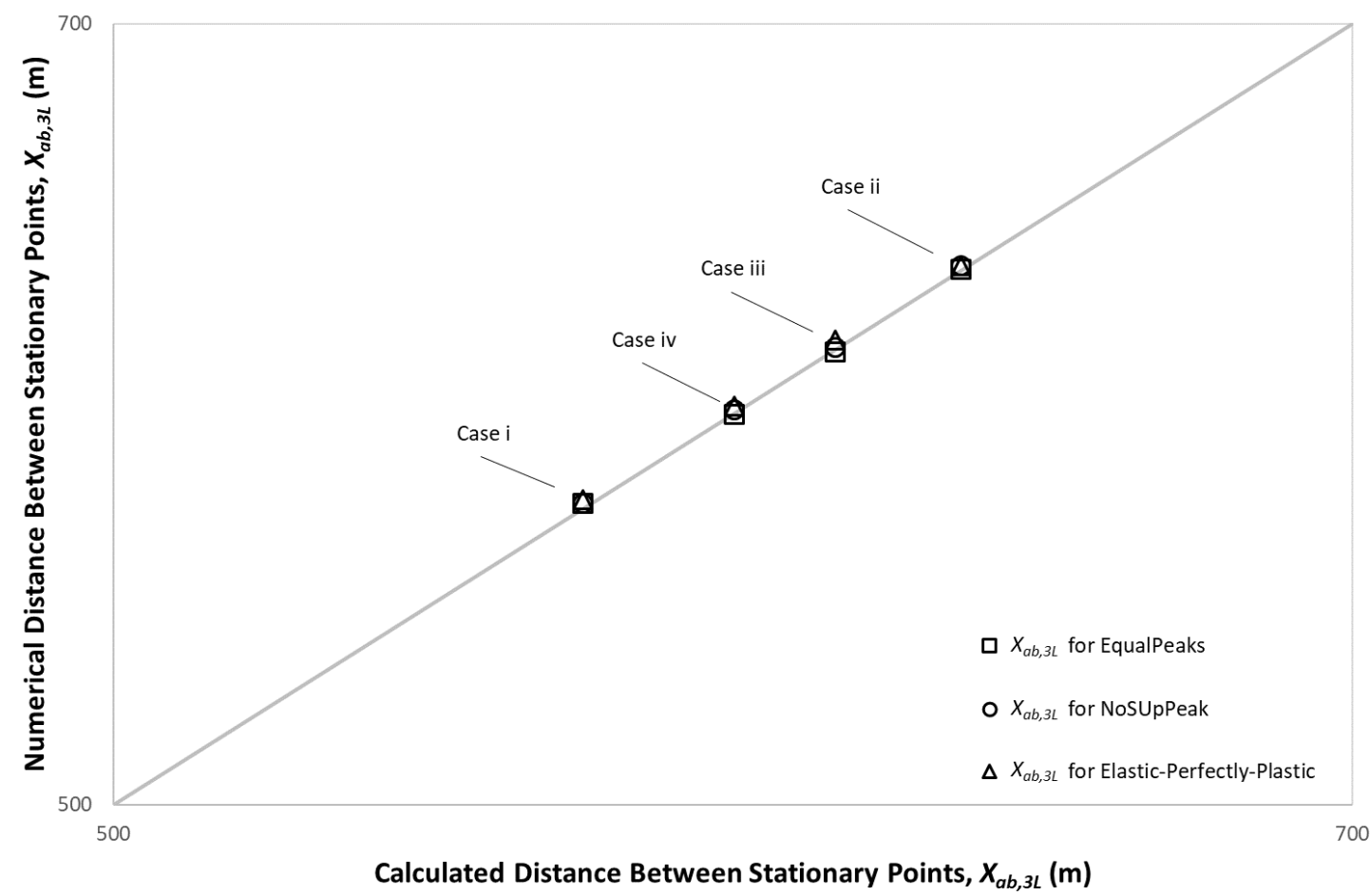

459

Calculated Distance Between Stationary Points, $X_{a b, 3 L}(\mathrm{~m})$

460

461 
Journal of Offshore Mechanics and Arctic Engineering

\section{Figure 12 Walking rate results}

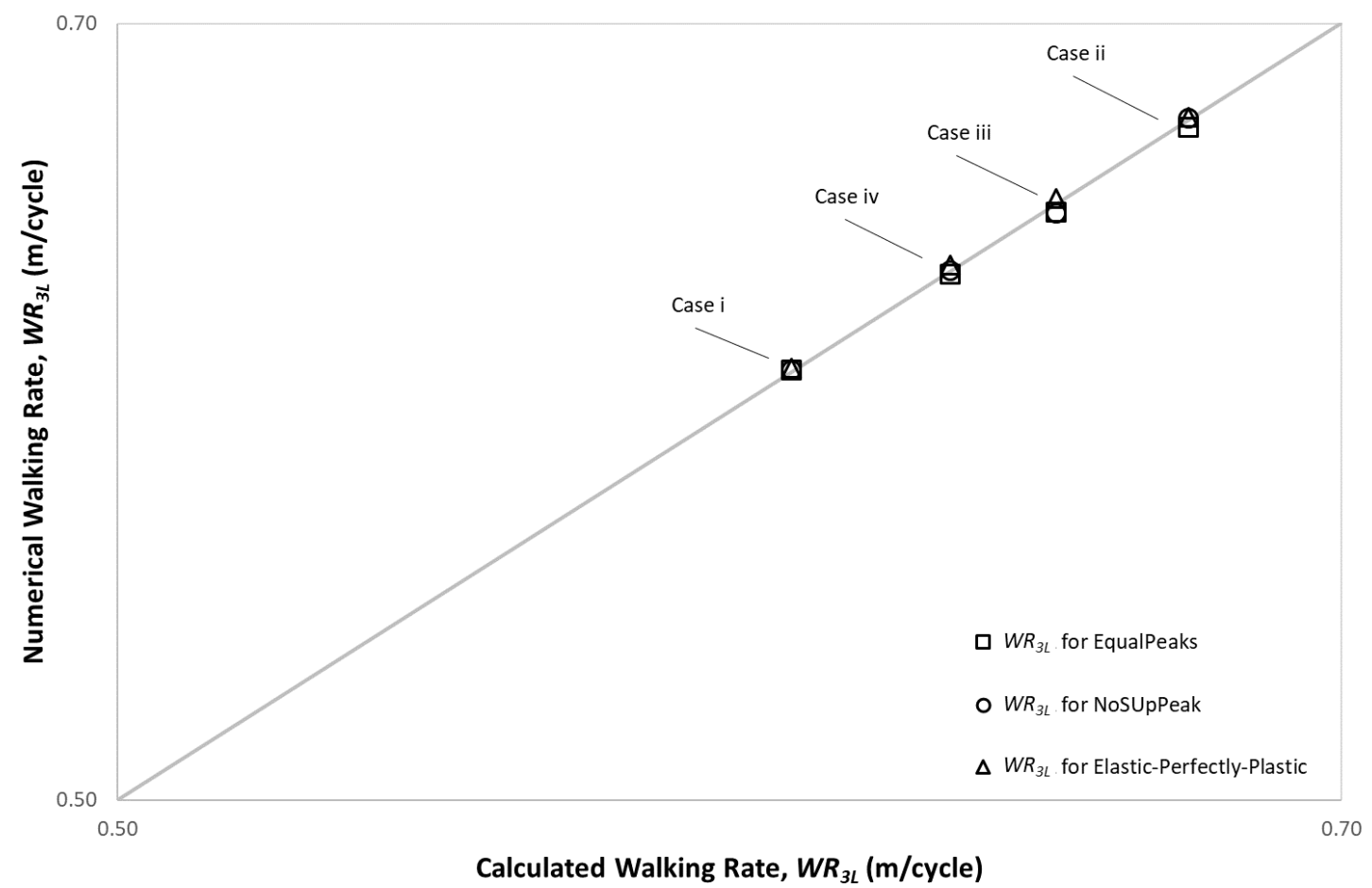

463

464

465 
Journal of Offshore Mechanics and Arctic Engineering

\section{TABLES}

467 Table 1 General properties

\begin{tabular}{ll}
\hline Parameter & Value \\
\hline Steel outside diameter, $O D$ & $0.3239 \mathrm{~m}$ \\
Steel wall thickness, $t$ & $0.0206 \mathrm{~m}$ \\
Length, $L$ & $5000 \mathrm{~m}$ \\
Seabed slope angle, $B$ & $2.0^{\circ}$ \\
Temperature variation, $\Delta T$ & $100^{\circ} \mathrm{C}$ \\
Pipe submerged weight, $W$ & $0.4 \mathrm{kN} / \mathrm{m}$ \\
Residual friction coefficient, $\mu$ & 0.25 \\
Steel Young's modulus, $E$ & $2.07 \times 10^{11} \mathrm{~Pa}$ \\
Steel Poisson coefficient, $v$ & 0.3 \\
Steel thermal expansion coefficient, $\alpha$ & $1.165 \times 10^{-5}{ }^{\circ} \mathrm{C}^{-1}$ \\
\hline
\end{tabular}

468

469 
Journal of Offshore Mechanics and Arctic Engineering

470

Table 2 Case properties

\begin{tabular}{|c|c|c|c|c|c|}
\hline \multirow{2}{*}{ Property } & & \multicolumn{4}{|c|}{ Cases } \\
\hline & & $\mathrm{i}$ & $\mathrm{ii}$ & iii & iv \\
\hline Peak Elastic Force, $F_{P}(\mathrm{kN})$ & & 0.200 & 0.400 & 0.300 & 0.250 \\
\hline $\begin{array}{l}\text { Peak Elastic Force } \\
\text { Displacement, } \delta_{\text {mobP }}(\mathrm{m})\end{array}$ & Mobilisation & 0.129 & 0.129 & 0.129 & 0.129 \\
\hline Residual Plastic Force, $F_{R}(\mathrm{kN})$ & & 0.100 & 0.100 & 0.100 & 0.100 \\
\hline $\begin{array}{l}\text { Residual Plastic Force } \\
\text { Displacement, } \delta_{m o b R}(\mathrm{~m})\end{array}$ & Mobilisation & 0.162 & 0.162 & 0.162 & 0.162 \\
\hline Ideal Mobilisation Displaceme & $\mathrm{t}, \delta_{m o b^{\prime}}(\mathrm{m})$ & 0.065 & 0.032 & 0.043 & 0.052 \\
\hline
\end{tabular}

471

472 
Journal of Offshore Mechanics and Arctic Engineering

473 Table 3 Tri-linear finite element analysis results for soil case ii

\begin{tabular}{lccc}
\hline Case & & Distance Between Stationary Points & Walking Rate \\
\hline \multirow{2}{*}{ ii } & EqualPeaks & $637 \mathrm{~m}$ & $0.674 \mathrm{~m} /$ cycle \\
& NoSUpPeak & $638 \mathrm{~m}$ & $0.675 \mathrm{~m} /$ cycle \\
\hline
\end{tabular}

474

475 
Journal of Offshore Mechanics and Arctic Engineering

476 Table 4 Rigid-plastic calculation results

\begin{tabular}{lcc} 
Case & Distance Between Stationary Points & Walking Rate \\
\hline Rigid-plastic (Carr et al., 2006) & $698 \mathrm{~m}$ & $0.740 \mathrm{~m} / \mathrm{cycle}$
\end{tabular}

477

478 
Journal of Offshore Mechanics and Arctic Engineering

479 Table 5 Analytical results

\begin{tabular}{lcc} 
Case & Distance Between Stationary Points & Walking Rate \\
\hline i & $576 \mathrm{~m}$ & $0.610 \mathrm{~m} /$ cycle \\
ii & $637 \mathrm{~m}$ & $0.675 \mathrm{~m} /$ cycle \\
iii & $617 \mathrm{~m}$ & $0.653 \mathrm{~m} /$ cycle \\
iv & $600 \mathrm{~m}$ & $0.636 \mathrm{~m} /$ cycle
\end{tabular}

480

481 
Journal of Offshore Mechanics and Arctic Engineering

482 Table 6 Tri-linear finite element analyses results

\begin{tabular}{|c|c|c|}
\hline Case & Distance Between Stationary Points & Walking Rate \\
\hline EqualPeaks & $577 \mathrm{~m}$ & $0.611 \mathrm{~m} /$ cycle \\
\hline NoSUpPeak & $577 \mathrm{~m}$ & $0.611 \mathrm{~m} /$ cycle \\
\hline EqualPeaks & $637 \mathrm{~m}$ & $0.674 \mathrm{~m} /$ cycle \\
\hline NoSUpPeak & $638 \mathrm{~m}$ & $0.675 \mathrm{~m} /$ cycle \\
\hline EqualPeaks & $616 \mathrm{~m}$ & $0.652 \mathrm{~m} /$ cycle \\
\hline NoSUpPeak & $617 m$ & $0.653 \mathrm{~m} /$ cycle \\
\hline EqualPeaks & $600 \mathrm{~m}$ & $0.635 \mathrm{~m} /$ cycle \\
\hline NoSUpPeak & $601 \mathrm{~m}$ & $0.636 \mathrm{~m} /$ cycle \\
\hline
\end{tabular}

483 